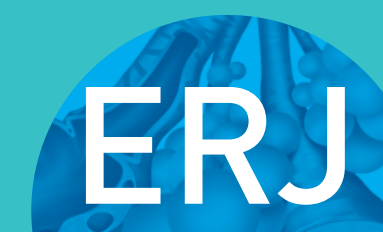

open research
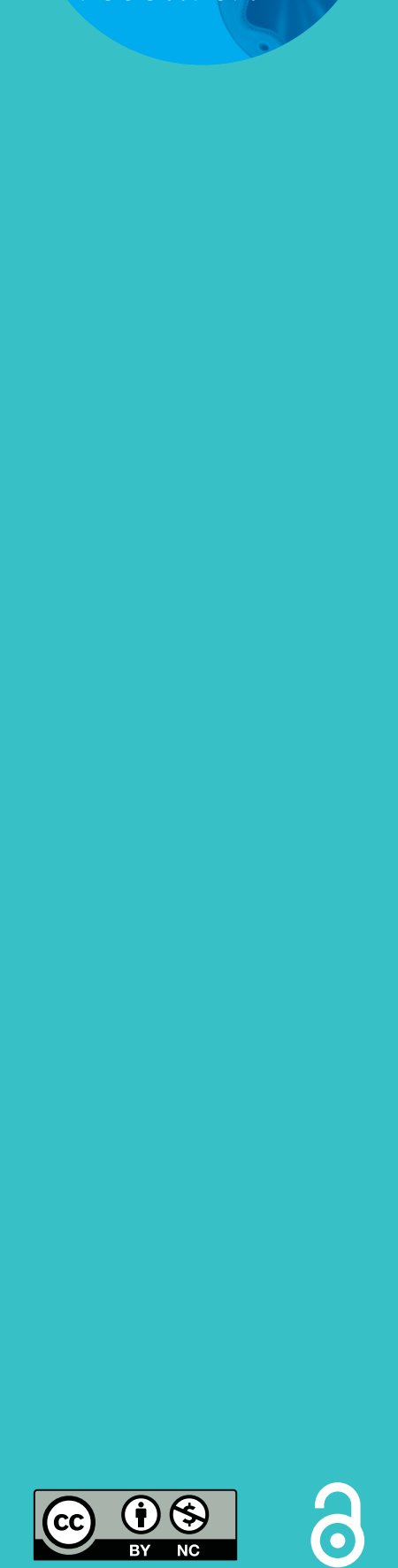

\section{Supporting patients self-managing respiratory health: a qualitative study on the impact of the Breathe Easy voluntary group network}

\author{
Ferhana Hashem and Rowena Merritt
}

Affiliation: University of Kent, Canterbury, UK.

Correspondence: Ferhana Hashem, Centre for Health Services Studies, George Allen Wing, Cornwallis Building, University of Kent, Canterbury, Kent, CT2 7NF, UK. E-mail: F.Hashemakent.ac.uk

ABSTRACT Self-management strategies are designed to improve lung and respiratory health through structured self-management plans with regular practitioner reviews. Strategies have not, however, focused upon how patient support groups and advocacy networks can help with the management of these conditions; therefore, it is unknown what impact they may have on patient self-management. A qualitative study was designed to help understand what impact the British Lung Foundation's Breathe Easy (BE) groups have on patients managing their lung and respiratory conditions.

A semistructured telephone interview schedule was developed to study the network. Topics covered included: perceptions about the BE groups; current referrals systems and integration pathways; benefits of attending the BE groups; and integration of the BE groups into the respiratory pathway.

Key themes explored included: shared patient experience and peer support; patient self-management and self-education; attendance of healthcare professionals; and the impact of integrating BE groups into the respiratory pathway.

BE networks were shown to support self-care initiatives for people attending the groups, and members expressed a social and educational benefit. BE networks were working with the local National Health Service to become an integral part of the respiratory pathway, yet there was evidence of resistance from the health service in incorporating the networks.

@ERSpublications

Lung and respiratory conditions affect $\sim 12.7$ million people in the UK with asthma, COPD or other longstanding respiratory illnesses; half are dependent on prescribed medicines. Voluntary patient support groups can provide peer support for self-management. http://ow.ly/7Xz330hbeth

Cite this article as: Hashem F, Merritt R. Supporting patients self-managing respiratory health: a qualitative study on the impact of the Breathe Easy voluntary group network. ERJ Open Res 2018; 4 : 00076-2017 [https://doi.org/10.1183/23120541.00076-2017].

This article has supplementary material available from openres.ersjournals.com

Received: June 222017 | Accepted after revision: Nov 142017

Support statement: This work was supported by the British Lung Foundation. Funding information for this article has been deposited with the Crossref Funder Registry.

Conflict of interest: None declared.

Copyright $\odot$ ERS 2018. This article is open access and distributed under the terms of the Creative Commons Attribution Non-Commercial Licence 4.0. 


\section{Introduction}

Respiratory conditions span a broad spectrum of disease groups: in some instances, the disease pathway for an individual can be terminal, and in other cases, improved disease management for patients can be key to the prevention of acute episodes [1]. In the UK, surveys of the general population suggest that $\sim 12.7$ million people (approximately one in five) have a history of asthma, chronic obstructive pulmonary disease (COPD) or another longstanding respiratory illnesses, and half of these ( $\sim .5$ million people) report taking a prescribed medication for lung disease in the last year [2]. For people with respiratory conditions, voluntary patient support groups can play a vital role in bringing together educational, social and practical advice for effective disease management $[3,4]$. This paper focuses on how patients can actively self-manage their condition through self-education initiatives and peer-support networks working alongside the collaboration of healthcare professionals (HCPs), all accessed via the UK's British Lung Foundation's (BLF) Breathe Easy (BE) voluntary group network.

Self-management is a concept that is becoming increasingly more salient and promoted through health policy initiatives to help people deal with the effects of long-term conditions (LTCs) and the economic implications associated with these. As a concept, self-management is notoriously difficult to define and there is no agreed definition of the term. SADLER et al. [4] suggest that wider definitions of self-management encompass social support from friends, family and wider community ties. It is also characterised by collaborative partnerships between patients and HCPs aimed at encouraging a relationship of care fostering self-management. In more commonly found respiratory diseases such as asthma and COPD, self-management involves formal patient education, written actions plans, self-monitoring by peak expiratory flow or symptoms, and regular medical review [5-8]. Although these are important features of a medicalised view of self-management, there are other models that have started to make inroads in which lay leaders initiate self-education programmes with the cooperation of HCPs; thus, an alternative model is emerging with patients taking the lead in self-management. BE groups provide a network of people-led self-management support:

Groups promote self-care via peer support, education and information giving. Integral to the success of the network are passionate and community-based volunteers, who drive groups forward to increase the health and wellbeing of their attendees. Volunteers are responsible for key roles within groups, with healthcare professionals working in partnership with some groups, providing advice, talks and establishing effective referral pathways [9].

Thus, a key component of patient support groups and advocacy networks such as BE are the social networking function they serve to help members share their experiences, encourage patient self-education, and in recent times, link members based within patient organisations to the local National Health Service (NHS) trusts [10]. In the case of BE groups, this has involved regular input by a HCP in which either a local respiratory nurse attends, the groups receives a talk from a clinician about respiratory health, or through a pulmonary rehabilitation taster session given by physiotherapists or occupational therapists. However, the type and level of clinical input can vary from month to month and from group to group, and not at all times were talks always delivered by HCPs. The overall function of the BLF's BE networks is to increase awareness of how members can manage their conditions through monthly educational talks, thereby opening up learning initiatives for disease management, whilst at the same time, the BE networks are becoming a core and integral part of a patient's treatment pathway, helping patients to self-manage and recognise their needs. Yet, the impact of peer support and social networking, as well as evidence on the effect of lay-led self-management on patients' health, is limited.

Published systematic review evidence has shown that there is little research on how patient support groups, advocacy networks or peer support can help with the management of lung/respiratory conditions. Rather, this body of literature has a much wider patient focus on those with mental health conditions, cancer support networks and beyond, but very limited evidence on managing long-term lung health. In the 2007 systematic review on lay led self-management education programmes in chronic conditions by FOsTER et al. [11], it was found that lay-led self-management programmes may lead to small, short-term improvements in patients' self-efficacy, self-rated health and cognitive symptom management, and an increase in frequency of aerobic exercise; yet, whilst there were small improvements, the improvements were not clinically significant. The review recommended that further research was needed to explore longer-term outcomes and the potential for lay-led self-management [11].

Recent literature on patient self-education and disease management have, however, been primarily in terms of structured self-management education involving information on which medicines they should take and when, prevention of symptoms and attacks, written action plans, and regular practitioner reviews [12-14]. The systematic reviews by Walters et al. [7], Gibson et al. [5] and Powell and Gibson [6] on patient self-management in COPD and in asthma found that self-management helped individuals recognise and react to the impact of an exacerbation, and taught patients the skills and behaviours they 
needed to manage their disease $[7,8,15]$. The studies recommended that self-management could have a positive impact if incorporated into a formal treatment pathway under the review of a practitioner. Yet, current systematic review evidence on patient self-education have been based in clinical settings under the guidance of a practitioner, rather than in less formalised settings based in patient support groups. It is therefore debatable whether the findings are relevant due to the lack of contextual overlap, as well as considerations around the impact of patient self-education programmes between peers or between patients and practitioners.

In recent years, there has been a gradual shift in the delivery, planning and organisation of healthcare in particular in patients with COPD, as well as other chronic respiratory diseases. In these disease groups, deterioration in lung function in some cases has increased acute and repeated exacerbations, use of polypharmarcy, increased visits to emergency departments, hospital admissions and length of stay, as well as the wider implication to patients' mental health and a steep decline in quality of life [16]. Many have argued that there is a need to rethink how respiratory services can be delivered outside traditional organisational boundaries in order to coordinate care between different services and ultimately to "improve processes and outcomes of care whilst making the most efficient use possible of scant healthcare resources". There is growing evidence to suggest the potential of community-based integrated care interventions to impact positively on disease knowledge and self-management [16].

The British Thoracic Society (BTS), an organisation representing professionals responsible for respiratory medicine and at the forefront of guideline production for the UK's National Institute for Health and Care Excellence, supports the rationale for integrated care in respiratory health. In 2015, the BTS recognised the value of integrated working, thereby proposing a definition of integrated care:

Integrated Care is the organisation and management of a health service such that people receive safe and effective care by the most appropriate health professional at the optimal time in the most appropriate environment. Such care should be offered with a patient centred and compassionate focus and achieve the desired results in a cost-effective manner. The integration of care should involve healthcare, whether hospital centric or within the community, and social care [17].

Notwithstanding, HCPs support efforts to encourage collaborative working through integrated care. PATEL [16] argued that there is a need to bridge integrated healthcare within community services that provide social support and address issues around preventative strategies in lung health. The BLF has been driving forward efforts to integrate BE groups into the local NHS, therefore joining up patient support groups and advocacy networks within local community respiratory teams, an example of which can be seen in the collaboration established between the local NHS and a BE group in the West Midlands. In 2011, NHS Stoke on Trent and North Staffordshire BE group put forward a model to increase collaborative working as part of the NHS Lung Improvement Programme. Some of these strategies included: establishing which general practitioner (GP) surgeries did and did not refer to the group, and working alongside practices to raise awareness of the potential impact of peer support; HCPs from the specialist community respiratory service being identified to input into BE group meetings to answer questions and offer additional advice; and lastly, the community respiratory team was involved in an official launch and raising awareness of the $\mathrm{BE}$ group with HCPs by running a workshop [18]. The rationale and key components of integration seem to offer a pathway of care that provides optimal benefits to patients in terms of the identification and delivery of respiratory care.

The purpose of this paper is to explore how BE groups open up avenues for patients to share their experiences and provide peer support, increase opportunities for raising awareness for patient self-education, and enable self-management through a key relationship via HCP facilitation. The onward results and analysis explore this component further. In addition, the study considers whether the steps towards BE groups being integrated into local respiratory teams have been implemented in the respiratory treatment pathway.

\section{Methods}

\section{Sampling strategy and recruitment}

Theoretical purposive sampling was used to select the participants, who were chosen according to predetermined criteria relevant to the study's key research objectives [19]. The sample was recruited within three main groups. The first comprised BLF volunteers who were members of the local BE group, some of whom held the position of Chair, Treasurer or Secretary, or were ordinary members, and in general, whose respiratory health was sufficiently stable to be a proactive and regular member of the group, but consequently, it was these members who were able to commit to a telephone interview, and therefore their involvement and proactive role would have impacted on their qualitative statements and responses. In some cases, ethically, it would not have been possible to invite all members, as many of them were ill with 
respiratory diseases in the long term. The participants were drawn from Standard BE groups (existing BE groups that had not been integrated into the local NHS pathway), Converted Integrated BE groups (existing BE groups that had been subsequently integrated into the local NHS pathway) and New Integrated BE groups (new groups set up specifically to be integrated into the local NHS pathway). The second group of participants was comprised of HCPs and clinical commissioning group (CCG) representatives, and the third groups of participants was composed of BLF steering group (SG) members. The research team ensured that all relevant groups were invited to take part and that different perspectives were included in the sample [20].

The participants were selected from a purposive sample. The BLF's integration management team initially approached potential participants to invite them to an interview and the participants then agreed in principle to be contacted by University of Kent (Canterbury, UK) researchers. The researchers at the university approached 42 people by e-mail or telephone and in total, 30 participants agreed to be interviewed (table 1). If the researchers did not hear back from the participants they were re-contacted on two more occasions and if there was still no response, then it was decided not to contact them anymore. No financial incentives were offered to participants.

\section{Methodological approach}

A qualitative approach was chosen, as this seemed the most appropriate in order to gather data from participants in their natural setting as we were able to talk to people through an interview over the telephone in an informal manner. As the research was concerned with people's views on the BE groups, it was important to capture the interactions, behaviours and meanings they attached to the groups; this approach is interpretive, which means that the researcher frequently has to make sense of assumptions or ideas that are taken for granted [21]. The underlying ontological position raises questions about what is the nature of the social world and what there is to know about it, so in our research, when gathering data, we saw the social world as open and subjective with participants shaped by agency and choice about what they did. In addition, our research also raised epistemological questions about knowing and learning, how we can learn about reality, and what is the basis of knowledge; we therefore used inductive logic to build our knowledge from the bottom up based upon our observations and understanding of the world from the people we interviewed drawing from their everyday lives [20].

\section{Conduct of interviews}

The participants were interviewed over the telephone once between July and December 2014. All interviews were recorded digitally. The interviews varied in length from 21 to $52 \mathrm{~min}$, with a median length of $38 \mathrm{~min}$. The participants were asked for their permission to record the interview. Written consent was also taken from the participants in advance. A list of topics and questions included in the interviews is given in the supplementary material.

\section{Data analysis}

The interviews were transcribed verbatim by the research team. The data were analysed using thematic analysis, which took place in four key steps. 1) Familiarisation, involving data immersing, gaining an oversight of the content and identifying topics of interest. 2) Constructing an initial thematic analysis involving refining and categorising the data into themes and subthemes. This process was assisted by using NVIVO (Qualitative Software and Research Pty Ltd, Doncaster, Australia). 3) Reviewing data extracts, which involved organising the data to work out if there were more coherent groups of data. 4) Summarising and writing up the data, which involved putting the data together using summaries for each of the groups with the summaries appearing for each theme and group of participants [20].

Then samples of the data were double-coded, and discussed between the research team to enhance reliability and construct validity [22]. At the analysis stage, the consistency of the findings and dependability of the data were advanced by having two researchers (F. Hashem and R. Merritt) each coding a sample of data independently, who then met together to come to an agreement on the emerging

\section{TABLE 1 Type and number of participants interviewed}

BLF: British Lung Foundation; HCP: healthcare professional; CCG: clinical commissioning group. 
themes and subthemes, and any discrepancies were overcome through a consensus discussion between them [23]. Any deviant themes were an indication that the researchers needed to consider additional information and restructure their understanding, which also contributed to refining the initial propositions, as well as help to reflect upon whether the original explanation applied in all circumstances [24]. Reliability and construct validity were also important factors for the study, as the researchers were concerned with analysing the results and ensuring the quality of the study, and furthermore, enhancing the dependability of the results by examining both the process and the product of the research for consistency [25].

The research team included two qualitative researchers both with over 10 years' experience of using qualitative approaches for collecting and analysing data.

\section{Ethical approval}

The study was approved by the University of Kent's School Research Committee based in the School of Social Policy, Sociology and Social Research, in 2014.

All informants gave written consent in advance to participate in the telephone interviews. Participants were sent the consent form by e-mail in the first instance or by post on request; consent was obtained in hard copy from nine participants and e-mailed consent was obtained from 21 participants.

\section{Results}

Shared patient experience and peer-support network

The BLF volunteers noted that the monthly meetings provided its members an invaluable forum for discussion and an ideal opportunity for sharing patient experience:

Some of them...they wouldn't go out...it got them out...with two or three of the people there, that's probably the only thing they've done in the last 6 months, a massive benefit...we try and balance it between education and social events.

BLF volunteer with severe asthma; BE group 01

We had a lovely day out so that they can interact with each other and talk generally about how they are managing... but they find coming to the group they get a better understanding about their lungs.

BLF volunteer with asthma; BE group 05

One vital element the BE groups provided was the support made available not just to people with lung and respiratory conditions but also to a wider, extensive group of friends and family, which helped to increase their understanding of the disease as well as bringing them into the fold of the support network:

$\mathrm{BE}$ is for people in a similar state, or their carers or friends, because we have a variety of people in our group... friends, family and patients themselves, because that's what a network or group is made up of...I'm quite passionate about these things because it will help people to have a better quality of life.... and yet it's difficult for them to be connected up to it.

BLF volunteer with COPD; BE group 11

The BE groups provided an extensive local network to those affected by respiratory and lung diseases, as well as to a broader spectrum of friends and family, which offered peer support, helping to share patient experience as well as providing a social networking mechanism to its members. The BLF SG members acknowledged the value of peer support offered to people at the monthly group meetings:

You can't underestimate the benefit of people being in the same boat as you...the health professional thing is all very well...attendees talk to each other and they see other people perhaps using oxygen or perhaps stopping for a breather or coughing a lot and they empathise with each other.

BLF SG; BE group 07

It was recognised that having members who were in the same position and being able to identify with other people in a similar situation was key to coping and self-managing their respiratory health.

\section{Patient self-management and self-education}

The BLF volunteers explained how they found joining a BE group helped in managing their condition:

Health management means people like me doing more for themselves than were done before...it's improved the quality of my life...it's well worth it to me. 
I went to a couple of them and thought how good they are... in an area to have that support...a support group with people with similar conditions to support each other... coping strategies, they pass that on.

BLF volunteer, former respiratory nurse; BE group 17

The BLF volunteers spoke about how attendance of the group helped to improve their quality of life, aided in developing coping strategies when they had an exacerbation, and was important in keeping them informed about new inhaler techniques:

[Name of group attendee stated] will turn up...it's winter months. She will do a refresher on inhalers...and the group love her because she talks their language...[she's] been coming in a good couple of years now and the difference is unbelievable.

BLF volunteer with sarcoidosis; BE group 03

These two BLF volunteers discussed how they were adapting and transforming their lives to help self-manage their condition as a result of joining their local BE group:

I do my breathing exercises...I find that...along with the medication that I'm on. And what I've learned from the physiotherapists coming and giving us talks, how to breathe using proper breathing techniques...benefits me personally but also it is helping the rest of the members of the group.

BLF volunteer with asthma; BE group 05

Talking to people and learning to be happy with other people...when my wife first died...and I got this condition...you just lock yourself away and you don't realise just how low you get...general things...not being lonely and try and be as happy as you can with other people. And I can tell them how I feel...it's done me a great favour as I'm busier than I've ever been...I'm not sitting down moping...I've moved into a place where there's other people my own age...we mix together...it's really changed by life since I've got involved with the BE.

BLF volunteer, condition not stated; BE group 16

HCPs acknowledged the growing salience of the BE groups in helping people with lung conditions self-manage by increasing their understanding of the disease processes:

Anything that helps people engage with the disease process is of benefit...there are different organisations out there and $\mathrm{BE}$ is one of the better ones. It does help people self-help...the current model of care to treat people and kick them out isn't really empowering people to treat their own conditions...get people to start taking responsibility for themselves to some degree.

HCP, GP; BE group 27

The above respondent, a GP, felt that patients needed to be able to manage their own condition, in particular as this could bring greater patient empowerment and less dependency on the NHS.

\section{Involvement of HCPs}

At some of the BE groups, attendance of a HCP underpinned the self-management and educational ethos of the group, and gave it a strong identity that was closely aligned with the local respiratory pathway, rather than existing as a stand-alone peer-support network. The contribution of a HCP providing a themed talk at each meeting was an important "hook" to attract new members and retain a regular monthly turnout from its members:

It's a great help...with local clinicians, the [question and answer] sessions have been very successful...with specific advice from them...the consultant or the nurse will answer it...it's a good place to pick up ideas.

BLF volunteer with asthma; BE group 18

The above respondent spoke about how having a HCP available enabled them to raise questions and obtain advice about their condition, thereby potentially circumnavigating a visit to a GP and placing fewer demands of time and resources on the NHS. It is to be noted, however, that not all groups were regularly attended by a HCP, and talks that were organised were not always themed around self-management but were broader in focus, covering social interests as well.

In addition, the informants mentioned how important it was to have the involvement of HCPs in referring patients to BE groups: 
Then we had pulmonary rehab[ilitation] in [name of location stated] very early as there were links into the hospital... and a lot of referral from pulmonary rehab into the group...we've got a model for referral of the appropriate people to come to a group.

BLF volunteer with asthma; BE group 04

They weren't but they are now...but it's in the guidelines now...it's in there now that all surgeries need to have a leaflet and to promote BE groups...it is now integrated into the care pathway...but we've called it something else.

HCP, nurse; BE group 26

The above respondent noted that GPs and practice nurses had gradually started to refer patients to BE groups. However, referrals from them were still fairly limited as there was less interest in including the groups in the respiratory pathway by the GP surgeries:

I think it needs more work...formally...I'm not sure to be frank. I don't think they are [being referred], they give information, but they are not formally referred into them, no...something that will help patients and see tangible benefits...otherwise it will become a dead duck...It's about patients self-managing so they can see me less, what I really want is [fewer] people coming through my door.

HCP, GP; BE group 27

I don't want GPs involved, really, as they're rubbish at signposting. No, we are really bad! I struggle with it all the time...How do we really?... They need to go to one place...I think the best person is the patient...but then it's "how do they find out"...you need to have motivated patients and that's the problem.

BLF SG, GP; BE group 21

The two GPs above acknowledged that as professionals, GPs were notoriously bad at not referring patients to BE groups. In the first statement, the participant indicated that GPs needed more encouragement and information about the benefits of the groups, and in the second statement, this GP recognised that they had a poor reputation for signposting patients to support groups to assist with self-management.

\section{Integration into respiratory pathway}

What it is...is all the respiratory professionals...come regularly to the meetings...so in that respect it was already integrated, as we work closely with the hospital...team. BLF works with him [the consultant] in the community, they provide training to GPs and practice nurses... and then someone has an exacerbation they are fast tracked... because all of the information is known in the community service runs into the hospital, so if they turn up at [Accident and Emergency] they automatically get put onto a respiratory ward without any triaging.

BLF volunteer with asthma; BE group 04

The above respondent indicated that integration was about linking different healthcare services from primary, community and acute care with local community BE groups, which would enable patients to access appropriate points of the healthcare system without being referred inappropriately between services.

Nonetheless, there were opinions expressed by some respondents who felt that although integration was an aspiration, there were doubts raised about whether it could be fully realised:

Some of the larger CCGs, I think, are difficult structurally to get in and do work with and there's probably...in terms of offering them help...in some of them they are absolutely fine...they've got commissioners and acute services and are quite happy to have help. So it's just a complete jigsaw out there...COPD is on quite a lot of CCGs must do list...they'll get the amount of groups I'm certain...getting the meaningful sign up...meaningful integration between the CCGs, between the acute trusts...between physios and everything else...it might be difficult in areas where integrated working doesn't naturally exist.

BLF SG member; BE group 08

[Name of healthcare organisation] needs to unblock and un-funnel this pathway...I don't understand it...and I work within it...but I don't really understand how it works...I can refer patients to pulmonary rehab but the GPs can't...it's just nonsense...a lot of it has been around tariffs (the cost of it)...and so it goes on...it's not integrated at all...they try to chuck asthma and everything else into it...but the secondary care team doesn't have the skills to do that...there's no provision for long-term support of people with people other than with COPD. 
The respondents expressed how frustrated they were that even though there was commitment to integration and working together to offer a seamless respiratory pathway, organisationally, barriers to integration continued to exist across different NHS services. As indicated in the second statement, there was evidence of fragmented care where primary and secondary care teams did not work together to offer support in particular for people with restrictive and obstructive disease such as COPD.

\section{Discussion}

The qualitative interviews undertaken for this study revealed that the BE groups provided a central function to its members on a multiplicity of levels, which helped to bring together people with lung and respiratory conditions, connect them with a wider network of friends and family, cement the link with HCPs and bring about closer integration into the local respiratory pathway. The combination of these elements have placed patients as a key component in managing their care as active mediators of their own condition and that of their peers. A summary of the impact of the BE group network is shown in figure 1.

A key finding was the role social networks played in providing a mechanism for members to share their experiences and provide peer support to other members of the BE group. In their examination of the role of social networks in LTCs, Rogers et al. [26] noted how "these forms of support are sometimes less obviously health related according to traditional definitions but nevertheless give a sense of purpose, belonging, and well-being for people with LTCs". It is noteworthy that there are parallels with the examination by ROGERS et al. [26] of social networking and peer support in LTCs with the wider definition of self-management by SADLER et al. [4]. Nonetheless, there is one key difference between the two with SADLER et al. [4] drawing importance to the salience of the collaborative partnerships between patients and HCPs as conducive to a relationship of care fostering self-management. ReEves et al. [3] remarked how salient social networks were in managing LTCs. They noted that these networks were adaptable and responsive to the levels of health need, and the overall support of the network was valued to a greater

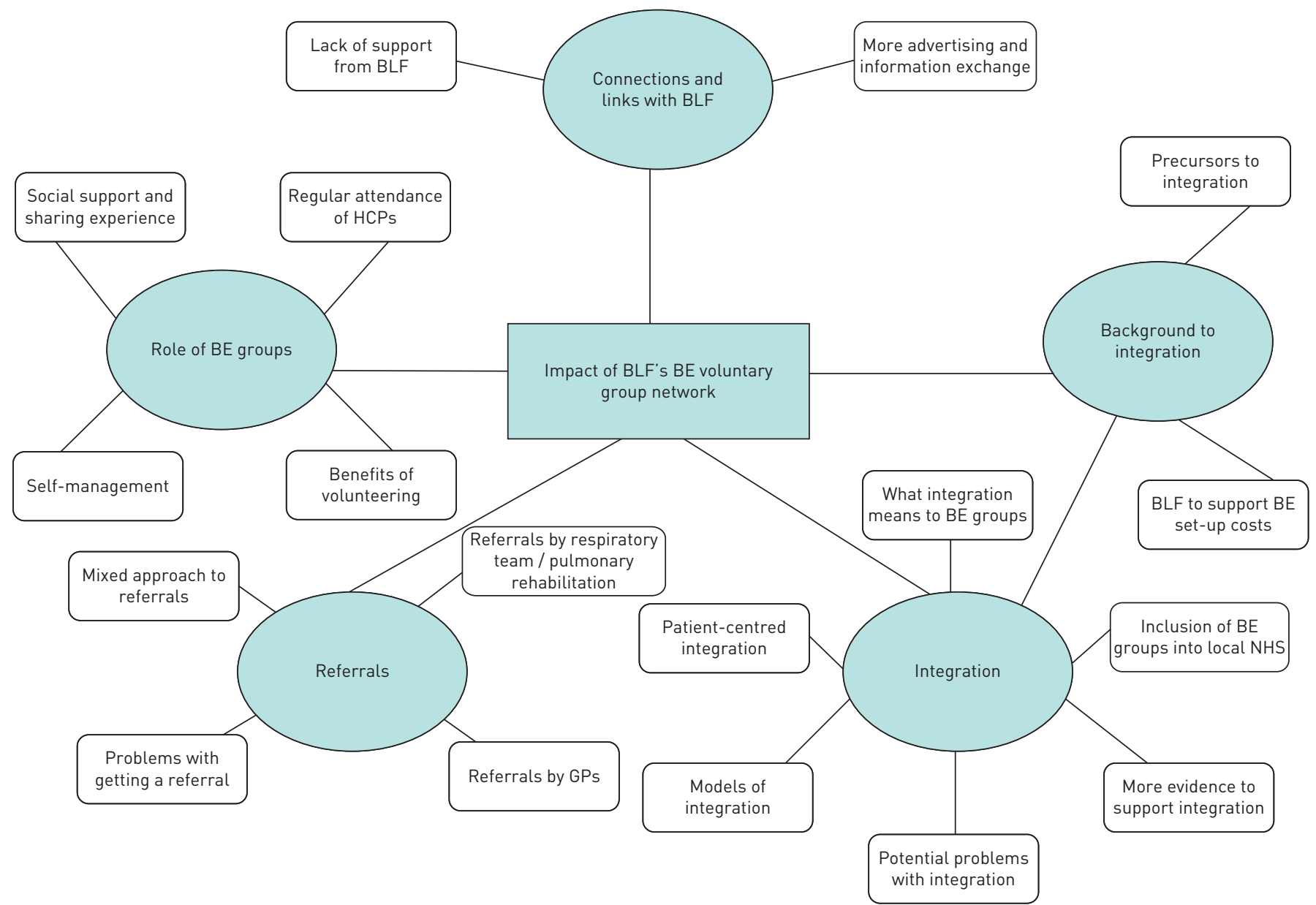

FIGURE 1 A summary diagram of the impact of the British Lung Foundation's (BLF) Breathe Easy (BE) voluntary group network. HCPs: healthcare professionals; GPs: general practitioners; NHS: National Health Service. 
extent than the relationship with individual members; in addition, ReEves et al. [3] found the altruistic and reciprocal nature of activities provided additional health benefits. Yet, is it noteworthy in the literature that the model of shared experience and peer support advocated by such networks was sometimes an unwelcomed source resisted by some patients [27]. This was not, however, found in our study.

In a recent systematic review of self-management between laypeople and HCPs, SADLER et al. [4] suggested that laypeople interpreted self-management as part of a broader construction of illness narratives, "enabling people to make sense of, cope with, and adapt to...their everyday lives", and reflected the quality of social support provided by their immediate social group, as these "informal networks [were] important in the process of coping". SADLER et al. [4] found that the HCPs conceptualised self-management as incorporating both a biomedical model of compliance and individual responsibility. These differences, although subtle, do help to explain why there are some key disparities of understanding in the model of self-management, and help to account for the limited amount of evidence on the effectiveness of self-management interventions [4]. The findings from our study showed that there were multiple perspectives on self-management from BLF volunteers, BLF SG members and HCPs. This resonates with the concept of self-management described by SADLER et al. [4], who talk about it encompassing a much wider definition from informal social support, to wider community ties as well as a partnerships with HCPs. What this means practically is that when incorporating self-management for respiratory diseases, the expectation of both the patient and practitioner may vary considerably and indicates there is a need to develop guidance on how self-management should work in both lay and clinical settings.

In terms of self-management interventions, as discussed above, the respondents expressed the importance of BE groups having strong links with local healthcare services via regular attendance of an HCP, which was purported to encourage self-management strategies. The increased contact with HCPs made available through the $\mathrm{BE}$ group meetings underlines the notion of self-management becoming central to addressing the burden of LTCs by "moving the day responsibility of managing health from the state to the individual, families and social groups" [28-30]. Given the complexity of self-management in respiratory diseases, regular contact with HCPs ideally should reduce acute admissions and thereby decrease the economic costs associated with it. The economic evaluation undertaken in the second phase of the study has shown a reduction in costs. The economic calculations included a benefit-cost analysis, social return on investment and a cost-effectiveness analysis, which showed a $42 \%$ reduction in unplanned GP visits and a $57 \%$ reduction in unplanned hospital admissions in Integrated BE groups compared with Standard BE groups [9]. Mossabir et al. [28] also noted that voluntary and community-based interventions impacted on health service use in reducing primary care contacts, a decrease in social issues brought up at consultations and fewer referrals of patients to other services, thus suggesting that community resources and equivalent social networks provide a linking role between healthcare providers and people with LTCs, which can in fact lead to a more efficient use of NHS services.

Referrals from HCPs to attend BE groups did take place; however, it was noted by the informants that there was an element of resistance from GP surgeries to promote the groups. In two review papers, the barriers encountered in GP surgeries to refer patients with LTCs to peer-support networks and voluntary organisations was a well-recognised phenomenon. SADLER et al. [4] observed that GPs believed that self-management conflicted with traditional healthcare practices and professional responsibility to patients; in addition, it was found that GPs/nurses and patients were keen to maintain the traditional professional-patient boundaries, and sidelined any discussions around self-management. MossaBIR et al. [28] also found in their review that clinicians were apprehensive about referring to voluntary organisations or were inappropriately referring patients to peer-support networks. The lack of enthusiasm and interest for making referrals from GP surgeries in our study showed a pattern of opposition and disregard for the salience of social networks and voluntary groups, as evident in previous studies.

The findings demonstrated that in at least some local health services, there was growing evidence of integration taking place to improve coordination of the respiratory pathway. Yet, there were statements from other informants indicating that clinical and organisational differentiation continued to lead to fragmented care. In their study on interorganisational integration in the Danish healthcare system, LyNGSø et al. [31] remarked how reforms and initiatives to integrate services did not alter how physicians and other staff collaborated across organisations. LYNGSø et al. [31] argued that one key issue was the concept of "integrated care", which was interpreted differently on many levels; they argued that "the lack of conceptual clarity is a major barrier to promoting integrated care, greatly hampering systematic understanding, successful real-world application and meaningful evaluation". They identified that the barriers and facilitators to integrated care related to themes including clinical, functional and normative integration, which were fundamental to developing integrated care in practice from the perspective of the HCPs [31]. The lack of conceptual clarity was also evident in our study, whereas some BLF volunteers spoke about integration as a much looser arrangement between different sectors of the health service and 
local BE groups, but principally being led by the local NHS respiratory teams, whilst other BLF volunteers thought integrated care was something that would be tightly coordinated under the local hospital consultant in the area. Hence, as in the study by Lyngsø et al. [31], definitions of integrated care varied considerably and perhaps have suffered not being adopted by the local NHS due to the lack of joint understanding.

\section{Conclusion}

This study shows that people with respiratory and lung conditions see a social benefit from attending BE groups, as they are able to share their experiences and gain peer support from this social network. We encountered BE group members who were adapting and transforming their lives to self-manage their condition and, through self-education, to understand their disease. There was evidence that GP surgeries were resistant to referring patients to $\mathrm{BE}$ groups, and ongoing efforts to encourage integrated respiratory care was taking place but this was patchy and differed across different NHS localities. Since the end of the study in 2016, national initiatives have now been put into place to encourage further integration of $\mathrm{BE}$ groups into the NHS, and there has been some headway in BE groups working alongside the NHS at the primary care level in CCGs.

\section{References}

1 Kruis AL, Smidt N, Assendelft WJ, et al. Integrated disease management interventions for patients with chronic obstructive pulmonary disease. Cochrane Database Syst Rev 2013; CD009437.

2 British Lung Foundation. Lung disease in the UK - big picture statistics. https://statistics.blf.org.uk/ lung-disease-uk-big-picture Date last accessed: October 17, 2017.

3 Reeves D, Blickem C, Vassilev I, et al. The contribution of social networks to the health and self-management of patients with long-term conditions: a longitudinal study. PLoS One 2014; 9: e98340.

4 Sadler E, Wolfe CDA, McKevitt C. Lay and health care professional understandings of self-management: a systematic review and narrative synthesis. SAGE Open Med 2014; 2: 2050312114544493.

5 Gibson PG, Powell H, Wilson A, et al. Self-management education and regular practitioner review for adults with asthma. Cochrane Database Syst Rev 2002; CD001117.

6 Powell H, Gibson PG. Options for self-management education for adults with asthma. Cochrane Database Syst Rev 2002; CD004107.

7 Walters JA, Turnock AC, Walters EH, et al. Action plans with limited patient education only for exacerbations of chronic obstructive pulmonary disease. Cochrane Database Syst Rev 2010; CD005074.

8 Zwerink M, Brusse-Keizer M, van der Valk PD, et al. Self management for patients with chronic obstructive pulmonary disease. Cochrane Database Syst Rev 2014; CD002990.

9 Merritt R, Pellat-Higgins T, Nizalova O, et al. Evaluation of the benefits of the British Lung Foundation's Integrated Breathe Easy voluntary group network: findings from the outcome, impact and economic evaluation. https://www.blf.org.uk/sites/default/files/IntegratedBreatheEasy-081216a.pdf Date last accessed: October 17, 2017. Date last updated: 2016.

10 Moreira T. Understanding the role of patient organizations in health technology assessment. Health Expect 2015; 18: 3349-3357.

11 Foster G, Taylor SJ, Eldridge S, et al. Self-management education programmes by lay leaders for people with chronic conditions. Cochrane Database Syst Rev 2007; CD005108.

12 Gatheral TL, Rushton A, Evans DJ, et al. Personalised asthma action plans for adults with asthma. Cochrane Database Syst Rev 2017; CD011859.

13 Toelle B, Ram FS Written individualised management plans for asthma in children and adults. Cochrane Database Syst Rev 2011; CD002171.

14 Roy A, Schultz TJ, Carson KV, et al. Asthma self management education with either regular healthcare professional review or written action plans or both in adults. Cochrane Database Syst Rev 2011; CD009479.

15 Howcroft M, Walters EH, Wood-Baker R, et al. Action plans with brief patient education for exacerbations in chronic obstructive pulmonary disease. Cochrane Database Syst Rev 2016; CD005074.

16 Patel I. Integrated respiratory care: what forms may it take and what are the benefits to patients? Breathe 2010; 6 : 253-259.

17 BTS Integrated Care Working Party. Working definition of Integrated Care. https://www.brit-thoracic.org.uk/ document-library/delivery-of-respiratory-care/integrated-care/working-definition-of-integrated-care-january-2015/ Date last updated: January 2015.

18 NHS Improvement. Managing COPD as a Long Term Condition: Emerging Learning from the National Improvement Projects. www.reedyfordgp.com/wp-content/uploads/2015/10/LungChronicManagement1-NHSImprovement-report-2011.pdf Date last accessed: October 20, 2017.

19 Guest G, Bunce A, Johnson L. How many interviews are enough? An experiment with data saturation and variability. Field Methods 2006; 18: 59-82.

20 Ritchie J, Lewis J, McNaughton Nicholls C, et al. Qualitative Research Practice: A Guide for Social Science Students and Researchers. London, NatCen, 2014.

21 Pope C, Mays N, eds. Qualitative Research in Health Care. Oxford, Blackwell Publishing Ltd, 2006.

22 Dye JF, Schatz IM, Sout N, et al. The qualitative report constant comparison method: a kaleidoscope of data recommended APA citation constant comparison method: a kaleidoscope of data. Qual Rep 2000; 4: 1-10.

23 Baxter P, Jack S. Qualitative case study methodology: study design and implementation for novice researchers. Qual Rep 2008; 13: 544-559.

24 Bazeley P. Qualitative Data Analysis: Practical Strategies. London, SAGE Publications, 2013.

25 Golafshani N. Understanding reliability and validity in qualitative research. Qual Rep 2003; 8: 597-606. 
Rogers A, Vassilev I, Sanders C, et al. Social networks, work and network-based resources for the management of long-term conditions: a framework and study protocol for developing self-care support. Implement Sci 2011 ; 6 : 56.

27 Blickem C, Kennedy A, Jariwala P, et al. Aligning everyday life priorities with people's self-management support networks: an exploration of the work and implementation of a needs-led telephone support system. BMC Health Serv Res 2014; 14: 262.

28 Mossabir R, Morris R, Kennedy A, et al. A scoping review to understand the effectiveness of linking schemes from healthcare providers to community resources to improve the health and well-being of people with long-term conditions. Health Soc Care Community 2015; 23: 467-484.

29 Von Korff M, Gruman J, Schaefer J, et al. Collaborative management of chronic illness. Ann Intern Med 1997; 127: 1097-1102.

30 Bodenheimer T, Lorig $\mathrm{K}$, Holman $\mathrm{H}$, et al. Patient self-management of chronic disease in primary care. JAMA 2002; 288: 2469-2475.

31 Lyngsø AM, Godtfredsen NS, Frølich A. Interorganisational integration: healthcare professionals? Perspectives on barriers and facilitators within the Danish healthcare system. Int J Integr Care 2016; 16: 4. 